\title{
Population genetics of forest type of Trypanosoma congolense circulating in Glossina palpalis palpalis of Fontem in the South-West region of Cameroon
}

\author{
Gustave Simo $^{1 *}$, Pythagore Soubgwi Fogue ${ }^{1}$, Tresor Tito Tanekou Melachio ${ }^{2}$, Flobert Njiokou ${ }^{2}$, Jules Roger Kuiate ${ }^{3}$ \\ and Tazoacha Asonganyi ${ }^{4}$
}

\begin{abstract}
Background: Genetic variation of microsatellite loci is a widely used method for the analysis of population genetic structure of several organisms. To improve our knowledge on the population genetics of trypanosomes, Trypanosoma congolense forest and savannah types were identified in the mid-guts of Glossina palpalis palpalis caught in five villages of Fontem in the South-West region of Cameroon. From the positive samples of Trypanosoma congolense forest, the genetic diversity and the population genetic structure of these parasites were evaluated.

Method: For this study, pyramidal traps were set up during three entomological surveys and 3347 tsetse flies were collected, dissected and 1903 midguts collected. DNA was extracted from midguts and specific primers were used to identify Trypanosoma congolense forest and savannah. All Trypanosoma congolense forest positive samples were characterized with seven microsatellite markers.

Results: Microscopic examination revealed 25 (1.31\%) mid-gut infections with trypanosomes while the PCR method identified 120 (6.3\%) infections due to Trypanosoma congolense: 94 (78.33\%) Trypanosoma congolense forest and 28 (21.77\%) Trypanosoma congolense savannah. The trypanosome infection rates varied significantly between villages and years of capture. Menji recorded the highest infection rate (15.11\%); and samples captured in 2009 were more infected (14.33\%). The microsatellite markers revealed a genetic variability between Trypanosoma congolense forest populations of Fontem villages and 6.38\% of mixed infections due to different genotypes of T. congolense "forest type".

Conclusion: Our data on the population genetics play in favor of a clonal reproduction of this parasite. The microsatellite markers used here showed a low genetic differentiation and an absence of sub-structuration $\left(F_{\mathrm{ST}} \leq 0.0003\right)$ between Trypanosoma congolense forest populations of Fontem villages. However, the high $F_{\mathrm{ST}}$ value $\left(F_{\mathrm{ST}} \geq 0.3911\right)$ between samples of the Democratic Republic of Congo and those of Fontem villages indicates low migration rates between trypanosomes of these subpopulations.
\end{abstract}

Keywords: Animal African Trypanosomiasis, Trypanosoma congolense forest, Trypanosoma congolense savannah, Microsatellite, Population genetics

\section{Background}

Trypanosomiasis is a disease caused by a parasite of the genus Trypanosoma. In Africa, many pathogenic trypanosomes such as Trypanosoma congolense (T. congolense), Trypanosoma vivax (T. vivax), Trypanosoma simiae (T. simiae) and Trypanosoma brucei brucei (T. brucei

\footnotetext{
* Correspondence: gsimoca@yahoo.fr

'Molecular Parasitology and Entomology Unit, Department of Biochemistry, Faculty of Science, University of Dschang, Cameroon, PO Box 67, Dschang, Cameroon

Full list of author information is available at the end of the article
}

brucei) affect livestock. Most of these parasites are mainly transmitted by tsetse flies of the genus Glossina [1]. In sub-Saharan Africa, thirty-seven countries are affected by the Animal African Trypanosomiasis (AAT) or nagana, covering an area of about 9 million $\mathrm{km}^{2}$. This situation could partly be responsible for the malnutrition in Africa [2] because it is estimated that about 50 million cattle are exposed to the disease and US $\$ 35$ million doses of trypanocides are used per year to overcome this pathology [3]. Controlling AAT would thus permit an increase in benefit 
for the agricultural and breeding industry to US $\$ 4.5$ billion per year [4].

$T$. congolense is considered as the most pathogenic trypanosome species of animals [5]. During the last decades, several studies have been carried out in order to understand the epidemiology of the diseases caused by $T$. congolense as well as the pathological manifestations resulting from infections caused by this pathogen [6,7]. From these studies, important data were generated on the prevalence and the distribution of $T$. congolense in many parts of Africa [8,9]. Despite these data, important knowledge on the biology, the genetic diversity and the population genetics of $T$. congolense remain to be elucidated. For instance, the reasons explaining the diversity of outcomes that are observed during $T$. congolense infections are not well understood although hypotheses on the genetic variability of trypanosomes have been proposed. Compared to other trypanosomes like for instance $T$. brucei subspecies where several studies have been undertaken on their genetic characterization in order to understand the epidemiological importance of these parasites [10-12], little investigation has been undertaken on the genetic characterization of different types of $T$. congolense. Previous studies characterizing T. congolense by isoenzyme electrophoresis reported a clonal reproduction in these parasites [13]. Recently, Morrison et al. [14] used microsatellite markers and revealed a mating system within $T$. congolense savannah type circulating in animals of West Africa region. With the same microsatellite markers, Simo et al. [15] generated data that suggested clonal reproduction within the forest type of $T$. congolense circulating in domestic animals of the South-West region of Cameroon. Most studies that investigated the genetic variability of $T$. congolense populations were undertaken on trypanosome strains isolated or circulating in vertebrate hosts such as cattle, goat, sheep, dog, and pig $[14,16]$. Up till now, no investigation has been undertaken on the genetic characterization of $T$. congolense circulating in tsetse flies of different areas of Africa. For T. brucei infections for instance, several studies reported genetic differentiation between $T$. brucei that infects mid-guts and salivary glands of different species of tsetse flies of west, central and east Africa $[17,18]$. Investigating the genetic variability of $T$. congolense circulating in tsetse flies may enable us to improve our knowledge on the population genetics of these parasites, and improve our understanding of the transmission of $T$. congolense between villages, and between tsetse flies and different vertebrate hosts.

In this study, we undertook the identification of Trypanosoma congolense in the mid-guts of Glossina palpalis palpalis of Fontem in the South-West Region of Cameroon. Thereafter, microsatellite markers were used to characterize the forest type of $T$. congolense in order to improve our knowledge of the population genetics as well as the circulation of these parasites.

\section{Methods \\ Study area}

Fontem $\left(5^{\circ} 40^{\prime} 12^{\prime \prime} \mathrm{N}, 9^{\circ} 55^{\prime} 33^{\prime \prime} \mathrm{E}\right)$ is located in the Lebialem division of the South-West Region of Cameroon. In this forested region, the climate is of tropical humid type and the topography is made up of hills and valleys through which several high speed rivers flow. The main activities of the Fontem population are agriculture, palm oil extraction, animal husbandry and poultry farming at a small scale. The present study was carried out in 5 villages; Besali, Bechati, Folepi, Agong and Menji (Figure 1).

\section{Sampling of tsetse flies}

Tsetse flies were sampled during three entomological surveys that were conducted in November 2006 (end of the rainy season), April 2007 and April 2009 (end of the dry season). During each survey, pyramidal traps [19] were set in tsetse fly favorable biotopes (cocoa plantations, food crop farms, palm farms, oil extraction points, water points, roads and footpaths, homesteads, and pig sties). A total of 363 traps of which 140 in November 2006, 111 in April 2007 and 112 in April 2009 were set. For each of these traps, geographical coordinates were recorded with a Global Positioning System (GPS etrex, high sensitivity). Traps were visited twice a day during 4 days of capture in each village. Living tsetse flies were dissected in a drop of $0.9 \%$ saline solution using a stereo microscope and their mid-guts were examined under a light microscope (magnification $\times 100$ ) for the presence of trypanosomes. Between dissections, the forceps were sterilized by immersing them in a solution of 0.1 molar sodium hydroxide for one minute and then in distilled water for another minute. After the microscopic examination, each mid-gut was collected and put in a microtube containing alcohol. In the field, the microtubes were conserved at ambient temperature; and at $-20^{\circ} \mathrm{C}$ in the laboratory until DNA extraction.

\section{DNA extraction}

DNA was extracted from tsetse mid-gut as described by Simo et al. [20]. In the laboratory, alcohol was evaporated during $60 \mathrm{~min}$ in an $80^{\circ} \mathrm{C}$ oven. Subsequently, $300 \mu \mathrm{l}$ of Chelex $5 \%$ were added to each tube and the mixture vortexed for $10 \mathrm{~min}$. Thereafter, two rounds of incubation took place; the first at $56^{\circ} \mathrm{C}$ for $30 \mathrm{~min}$, and the second at $98^{\circ} \mathrm{C}$ for $60 \mathrm{~min}$. The tubes were then centrifuged at $14000 \mathrm{rpm}$ for $5 \mathrm{~min}$ and the supernatant (DNA extract) was collected and stored at $-20 \mathrm{C}$ or used directly for PCR. 


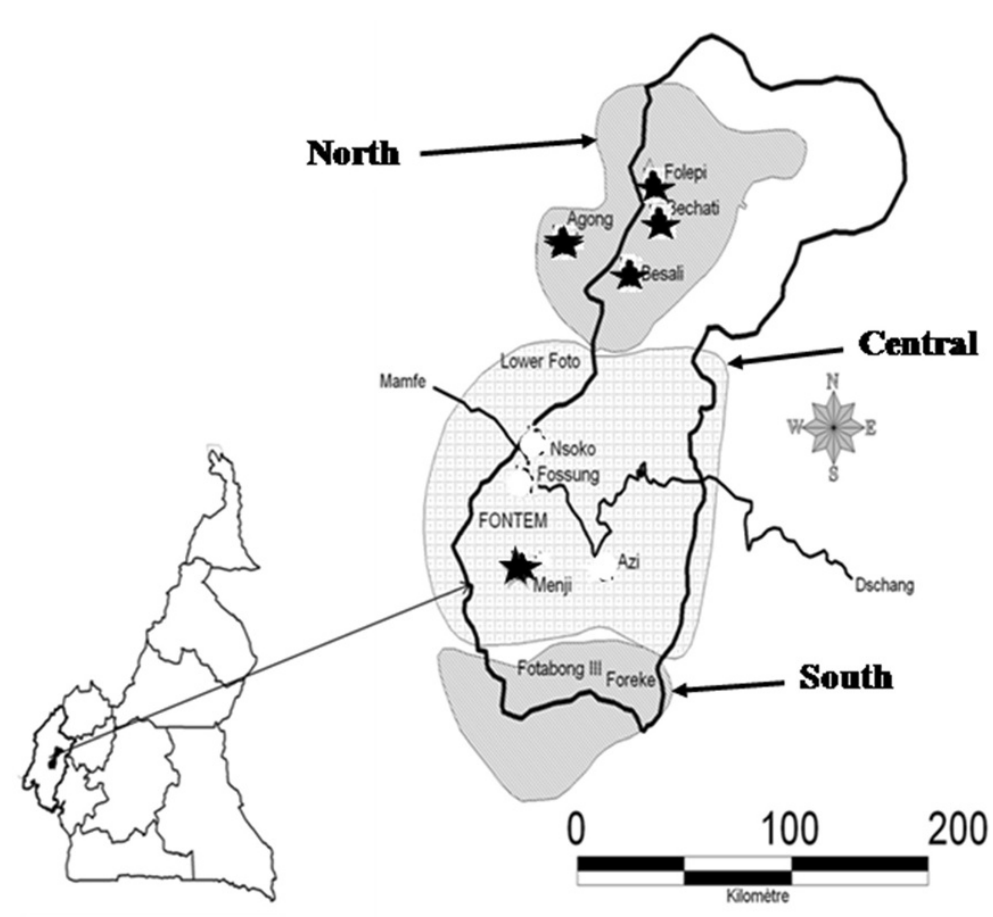

$\star$ Villages where tsetse flies were sampled

Figure 1 Map showing the villages of the Fontem where tsetse flies were sampled.

Identification of Trypanosoma congolense "savannah" and "forest" types

These trypanosomes were identified with specific primers: TCF1 (5' - GGACACGCCAGAAGTACTT-3') and TCF2 (5' - GTTCTCGCACCAAATCCAAC-3') for T. congolense forest [21]; and TCS1 (5'-TCGAGCGAGAACGGGCAC TTTGCGA-3') and TCS2 (5'-ACAATTAGGGACAAAC AAATCCCGC-3') for T. congolense savannah [22]. The amplification reactions were carried out as described by Herder et al. [23]. Each amplification reaction was performed in a total volume of $15 \mu \mathrm{l}$ containing 20 pmol of each primer, $10 \mathrm{mM}$ of Tris-Cl ( $\mathrm{pH} 8.3$ ), $50 \mathrm{mM}$ of $\mathrm{KCl}, 1.5 \mathrm{mM}$ of $\mathrm{MgCl} 2,1 \mathrm{mM}$ of each dNTPs, $3 \mu$ of DNA template and 1unit of Taq DNA polymerase. Amplification involved a pre-denaturation step at $94^{\circ} \mathrm{C}$ for 5 minutes followed by 40 amplification cycles made up of a denaturation step at $94^{\circ} \mathrm{C}$ for 30 seconds, a hybridization step at $60^{\circ} \mathrm{C}$ for 30 seconds, and an elongation step at $72^{\circ} \mathrm{C}$ for 1 minute. These were followed by a final elongation step at $72^{\circ} \mathrm{C}$ for $10 \mathrm{~min}$. The amplification products were resolved by electrophoresis at 100 volts for $30 \mathrm{~min}$ on $2 \%$ agarose gel containing ethidium bromide. DNA bands were visualized under ultraviolet light.
Genetic characterization of samples positive for the forest type of $T$. congolense

For this genetic characterization, all samples with a DNA sequence specific for the forest type of $T$. congolense were selected. For this characterization, the seven microsatellite DNA markers described by Morrison et al. [14] were used.

\section{Amplification of microsatellite DNA sequences}

For this study, each PCR was carried out in a final volume of $25 \mu \mathrm{l}$ containing $5 \mu \mathrm{l}$ of DNA extract and $1.5 \mathrm{mM}$ of $\mathrm{MgCl}_{2}, 20 \mathrm{pmol}$ of each primer, $1 \mathrm{mM}$ of each dNTP and 1 unit of Taq DNA polymerase. For each sample, two PCR rounds were performed for each marker as described by Morrison et al. [14]. During these PCR rounds, two different pairs of primers were used. The first PCR round was carried out with two primers as described by Morrison et al. [14]. The amplification cycles contain one denaturing step at $95^{\circ} \mathrm{C}$ for 5 minutes followed by 30 amplification cycles. Each cycle had a denaturation step at $95^{\circ} \mathrm{C}$ for 50 seconds, an annealing step at $52^{\circ} \mathrm{C}$ for 50 seconds, and an extension step at $72^{\circ} \mathrm{C}$ for 1 minute. This was followed by a final extension at $72^{\circ} \mathrm{C}$ for 10 minutes. For the second PCR round (nested PCR), a 1/10 dilution of amplified products of the first 
PCR round was used as template, and the set of primers used were designed from a region located between the primers used for the first PCR round. The second PCR was performed in the same conditions as the previous.

After the two PCR rounds, $7 \mu$ l of each PCR product were checked by electrophoresis on $2 \%$ agarose gels for $30 \mathrm{~min}$ at 100 volts. The allele bands were then resolved and their sizes determined on 10\% non-denaturing acrylamide gels. For agarose and acrylamide gels, bands were stained with ethidium bromide and visualized under UV light. After resolution of the amplified products on the polyacrylamide gel, a sample was defined as a multiple infection if it contained more than two alleles.

In addition to $T$. congolense forest positive samples of tsetse flies of Fontem, nine samples from Glossina fuscipes quanzensis of the Democratic Republic of Congo's were analyzed as above and subsequently included as out groups during the population genetics study.

\section{Genetic data analysis}

Population structure was assessed through Wright's F-statistics [24]. $F_{\text {IS }}$ measures the deviation from random union of gametes within subsamples and $F_{\mathrm{ST}}$ the deviation from random distribution of individuals between subsamples (and thus a measure of population differentiation). For the population structure analysis, all the individuals that displayed a single infection (78 individuals) were divided into four subpopulations according to their village of origin. Allelic richness was estimated with Fstat 2.9.4 software and tested through 10000 permutations within subsamples. Wright's F-statistics were estimated using Weir and Cockerham's unbiased estimators [25] in Fstat 2.9.4 software ([26] updated from Goudet [27]). The estimator used for $F_{\mathrm{IS}}$ was $f$ and its significance was tested through 10000 permutations of alleles within subsamples. For $F_{\mathrm{ST}}$, the estimator was $\theta$ and its significance was tested through 10000 permutations of individuals between subsamples. To get a general idea of individual distribution across the villages, an unrooted NJTREE was computed with MEGA 3.1 software [28] using the Cavalli-Sforza and Edwards [29] chord distances matrix, which were computed in the MSA software [30] and formatted in the software PHYLIP 3.69 [31].

\section{Statistical analyses}

The Pearson chi-square test was used to compare the trypanosome infection rates when $80 \%$ of theoretical values were higher than 5 . For the microscopic examination, the Pearson chi-square test with Yates correction was used to compare the trypanosome infection rates between years of capture because less than $80 \%$ of theoretical values were higher or equal to 3 and lower than 5 . When $80 \%$ of theoretical values were lower than 3, Fisher's exact test was used. These statistical analyses were performed using XLSTAT-PRO 3 software version 2009. The significant threshold was $5 \%$.

\section{Results}

\section{Entomological surveys}

During the three entomological surveys, 363 pyramidal traps captured 3347 tsetse flies belonging to G. p. palpalis subspecies. Of these flies, 1903 (56.85\%) of them that were still alive were randomly selected and dissected: 355 (72.15\%) of 492 in 2006, 1241(56.33\%) of 2203 in 2007 and 307 (47.08\%) of 652 in 2009. Of the 1903 dissected tsetse flies, microscopic examination revealed 25 (1.31\%) mid-gut infections with trypanosomes. The results of the microscopic examination according to villages and years of capture are reported in Table 1 . No significant difference was observed between microscopic examination results $(P=0.19318$ between villages; $P=0.246$ between years of capture).

\section{Molecular identification of the forest and savannah type of $T$. congolense}

The PCR targeting a multi-copy repeat sequence specific to trypanosome species revealed 120 (6.31\%) Trypanosoma congolense infections: $28(1.47 \%)$ were $T$. congolense savannah and 94 (4.93\%) T. congolense forest. Menji recorded the highest infection rate of $15.82 \%(44 / 278)$, followed by $5.15 \%$ (23/446) for Bechati and 4.52\% (52/ 1148) for Folepi (Table 1). No T. congolense savannah was identified at Agong. Comparing the infection rates between villages, there was significant difference for the forest type of $T$. congolense $(P<0.0013)$ and the savannah type $(P<0.00001)$ (Table 1$)$. A significant difference was also observed $\left(X^{2}=41.389 ; P<0.0001\right)$ when the infection rates of $T$. congolense (forest and savannah types) were compared between years of capture (Table 2). The highest infection rate was $14.33 \%(44 / 307)$ in 2009 , followed by

\begin{tabular}{|c|c|c|c|c|c|c|}
\hline \multirow[t]{2}{*}{ Localities } & \multirow[t]{2}{*}{ NC } & \multirow[t]{2}{*}{$\mathrm{NE}$} & \multirow[t]{2}{*}{$\mathrm{T}+(\%)$} & \multicolumn{3}{|c|}{ Number of positive PCR (\%) } \\
\hline & & & & TCF & TCS & NP \\
\hline Agong & 25 & 22 & $0(0)$ & $1(4.45)$ & $0(0)$ & $1(4.45)$ \\
\hline Besali & 40 & 9 & $0(0)$ & $0(0)$ & $0(0)$ & $0(0)$ \\
\hline Bechati & 683 & 446 & $10(2.24)$ & $19(4.26)$ & $4(0.89)$ & $23(5.15)$ \\
\hline Folepi & 2091 & 1148 & $10(0.87)$ & $45(3.92)$ & $8(0.69)$ & $52(4.52)^{* *}$ \\
\hline Menji & 508 & 278 & $5(1.8)$ & $29(10.43)$ & $16(5.75)$ & $44(15.82)^{* *}$ \\
\hline Total & 3347 & 1903 & $25(1.31)$ & $94(4.93)$ & $28(1.47)$ & $120(6.3)$ \\
\hline$P$ & & & $0.19318^{*}$ & $<0.0013$ & $<0.00001$ & $<0.0001$ \\
\hline
\end{tabular}

NC: Number of tsetse captured; NE: number of tsetse examined; TCF: Trypanosoma congolense forest; TCS: Trypanosoma congolense savannah; NP: number of tsetse flies with $T$. congolense (forest and/or savannah type) in their mid-guts; P: $p$-value; *P value not significant; ${ }^{* *}$ refer to villages where mixed infections were identified. 
Table 2 Infection rates of Trypanosoma congolense according to years capture

\begin{tabular}{lcccccc}
\hline $\begin{array}{l}\text { Years of } \\
\text { capture }\end{array}$ & NC & NE & T+ (\%) & \multicolumn{3}{c}{ Number of positive PCR (\%) } \\
\cline { 5 - 7 } & & & & TCF & TCS & NP \\
\hline 2006 & 492 & 355 & $1(0.28)$ & $12(3.38)$ & $0(0)$ & $12(3.38)$ \\
2007 & 2203 & 1241 & $18(1.45)$ & $42(3.38)$ & $22(1.77)$ & $64(5.16)$ \\
2009 & 652 & 307 & $6(1.95)$ & $40(13.03)$ & $6(1.95)$ & $44(14.33)^{* *}$ \\
Total & 3347 & 1903 & $25(1.31)$ & $94(4,93)$ & $28(1.47)$ & $120(6.36)$ \\
$X^{2}$ & & & 2.804 & 51.018 & 6.573 & 41.389 \\
$P$ & & & $0.246^{*}$ & $<0.0001$ & 0.037 & $<0.0001$ \\
\hline
\end{tabular}

NC: Number of tsetse captured; NE: number of tsetse examined; $T+$ number of tsetse flies found infected by the microscopy; TCF: Trypanosoma congolense"forest type"; TCS: Trypanosoma congolense"savannah type"; NP: number of tsetse flies with $T$. congolense (forest and/or savannah type) in their midguts; $X^{2}$ : Chi-square; $P$ : $p$-value; *P value not significant; ** refer to the year where mixed infections were identified.

$5.16 \%(64 / 1241)$ in 2007 and $3.38 \%(12 / 355)$ in 2006 . For the forest type of $T$. congolense, about $13.03 \%$ of dissected tsetse flies were infected in 2009 while $3.38 \%$ were infected in 2006/2007. There was a significant difference in the infection rates of $T$. congolense forest $\left(X^{2}=51.018\right.$; $P<0.0001)$ between capture years. For $T$. congolense savannah, no infection was observed in tsetse flies caught in 2006 . However, about $1.77 \%$ and $1.95 \%$ of tsetse flies caught in 2007 and 2009 respectively had mid-gut infections with $T$. congolense savannah; a significant difference $\left(X^{2}=6.573 ; P=0.037\right)$ was found for $T$. congolense savannah infection rates between these years of capture.

Two mixed infections of $T$. congolense forest and $T$. congolense savannah were found in two tsetse flies caught at Menji and Folepi in 2009.

\section{Genetic characterization of $T$. congolense forest type}

This characterization was performed only on the $94 T$. congolense forest positive samples. Of the seven microsatellites markers used in this study, no amplification was obtained for marker TCM3 while only $6.38 \%$ of these samples were amplified by TCM5 marker. These two markers were not considered for subsequent analyses. Out of the nine T. congolense forest samples from DRC, TCM5 amplified all of them, while TCM3 gave no amplification for these samples. For the five remaining markers, the amplification efficiency or their sensitivity differed considerably. The TCM7 marker showed the lowest amplification rate with $81.91 \%$ of $T$. congolense positive samples amplified, whereas the highest amplification rate was $94.68 \%$ for TCM4. TCM1, TCM2 and TCM6 amplified $86.17 \%, 85.10 \%$ and $90.42 \%$ of $T$. congolense positive samples, respectively. Details concerning the size of alleles at each microsatellite locus are reported in Additional file 1.

The five markers considered here have shown a genetic diversity in the population of $T$. congolense forest that circulates in tsetse flies of Fontem. This diversity varies according to microsatellite markers (Table 3). For instance, TCM2 was the most polymorphic marker with six alleles while TCM4 was the least polymorphic with only two alleles. For TCM1, TCM6 and TCM7, five alleles were identified for each of them. Some specific alleles such as 240 of TCM1, 190 of TCM2, 180 of TCM4 and 200 of TCM6 were found only in T. congolense forest of tsetse flies caught at Menji while the alleles 160 and 177 of TCM7 as well as 173 of TCM6 were found in parasites circulating in tsetse flies of Folepi and Bechati, respectively (Additional file 2).

All alleles identified in $T$. congolense of tsetse flies caught in 2006 were also found in parasites from tsetse flies sampled in 2007 and/or 2009. However, some alleles such as 240 of TCM1, 190 of TCM2, 180 of TCM4, 200 of TCM6 and 177 of TCM7 were identified only in T. congolense forest from flies sampled in 2009, while the alleles 173 of TCM6 and 208 of TCM7 were identified only in parasites of tsetse flies caught in 2007 (Additional file 3).

Our results show the highest value of the allelic richness of 2.896 for T. congolense populations identified in tsetse flies caught in 2009. In 2006 and 2007, the values of the allelic richness were 2.181 and 2.581, respectively. Comparing the allelic richness according to the years of capture, no significant difference $(P=0.0715)$ was observed. According to villages, the highest value of the allelic richness $\left(R_{S}\right)$ of 3.196 was observed for the population of $T$. congolense found in tsetse flies caught at Menji while the parasites identified in tsetse flies caught at Folepi and Bechati recorded $R_{S}$ values of 2.85 and 2.388 , respectively. No significant difference $(P=0.0655)$ was also found for the allelic richness between villages.

\section{Population genetics analysis}

Only single infections of $T$. congolense forest were taken into consideration for these analyses. We considered single infections as samples for which only one allele (homozygote) or two different alleles (heterozygote) were identified for all the five microsatellite markers used. In this context, a sample was considered as having multiple infections if it had more than two alleles for a given locus. TCM2 and TCM7 each revealed one mixed infection while four mixed infections were revealed by TCM1. In

Table 3 Number of allele and heterozygosity values at
each locus
\begin{tabular}{llllll}
\hline Loci & TCM1 & TCM2 & TCM4 & TCM6 & TCM7 \\
\hline N & 5 & 6 & 2 & 5 & 5 \\
Ho & 0.849 & 0.949 & 0.011 & 0.780 & 0.916 \\
Hs & 0.600 & 0.586 & 0.011 & 0.515 & 0.522 \\
\hline
\end{tabular}

$\mathrm{N}$ : Number of alleles at each locus; Ho: observed heterozygosity; $\mathrm{Hs}$ : expected heterozygosity. 
addition to multiple infections, all samples showing no amplification for at least three microsatellite loci were excluded for the population genetics analyses. In sum, 87 T. congolense forest samples with 78 from Fontem and 9 from DRC were used for the population genetics studies.

Multilocus genotypes, obtained for each $T$. congolense forest sample and for the five microsatellites markers considered here, enabled us to determine the level of genetic differentiation between the $T$. congolense forest populations. The genetic analyses performed on the 87 single infections of $T$. congolense forest type revealed low genetic differentiation between samples from different villages or different years of capture. To appreciate the genetic variability within the population of the forest type of $T$. congolense that circulates in tsetse flies of Fontem villages, the multilocus genotypes were used to construct a dendrogram of similarity by calculating Cavalli-Sforza and Edwards [29] chord distances for all pairwise comparisons (Figure 2). Thirty-four different genotypes were obtained for the 87 samples considered for these analyses, indicating inconsistent data with mating reproduction of particular genotypes. About 30 different genotypes were identified in villages of Fontem. However, the genetic distances between samples of Fontem were very low (Figure 2). The dendrogram obtained here can be subdivided into two clusters; the first cluster contains all samples of Fontem villages while the second cluster contains samples of the DRC. The population of $T$. congolense forest that circulates at Fontem seems to form a large single group with little genetic differentiation (Figure 2).

When the samples from different villages are considered as a single population, all the five loci exhibited heterozygote excess as shown by the global in breeding coefficient $\left(F_{\text {IS }}\right)$ of $-0.52818(\mathrm{P}<0.0001)$, which showed a predominant clonal reproduction of $T$. congolense forest (Figure 3 ). The pairwise $F_{\mathrm{ST}}$ values between subsamples indicate no genetic differentiation between samples from different villages of Fontem as well as samples from different years of capture (Table 4). However, the $F_{\mathrm{ST}}$ values obtained between subsamples of Fontem villages and those of the DRC show a greater genetic differentiation $\left(F_{\mathrm{ST}}\right.$ ranged from 0.3911 to 0.4212 ), and thus suggesting a very low migration rate of $T$. congolense between Fontem and DRC.

\section{Discussion}

The samples analyzed in this study allowed us to improve our understanding of the population genetics of the forest type of Trypanosoma congolense. Both microscopic examination and PCR detected trypanosomes in the mid-guts of tsetse flies of different villages of Fontem in the Southwest Region of Cameroon as already reported by previous authors [18,32]. The identification of $T$. congolense forest and savannah confirms the results obtained by previous authors who identified these parasites in tsetse flies [32] and domestic animals [15,33,34] of different villages of Fontem.

Our results show that $T$. congolense forest has a higher infectious rate than $T$. congolense savannah. The low infection of $T$. congolense savannah is probably due to the level of pathogenicity of this trypanosome subspecies. Indeed, Bengaly et al. [35] have shown that Zebu cattle infected by $T$. congolense savannah presented a severe syndrome, which led inexorably to death within four to seven weeks post-infection if no treatment was administered. This probably occurs in the Fonten area since the inhabitants of this area do not use trypanocidal drugs to prevent AAT. This would mean that the number of animals infected by $T$. congolense savannah and on which tsetse flies can become infected by this trypanosome subspecies would be considerably reduced. Moreover, the lack of trypanocidal drug to prevent AAT could explain the increasing trypanosome prevalence from one year to the next ( $T$. congolense infection rates have tripled from 2007 to 2009 and quadrupled from 2006 to 2009). These results indicate that for large scale breeding of animals in this area, control measures for AAT at Fontem need to be instituted.

Out of the seven microsatellite markers used in this study, only five gave good amplification for T. congolense positive samples from Fontem villages. These results are in line with those of Simo et al. [15] who reported identical results when they analyzed samples from domestic animals of the same villages. The fact that only few samples were amplified by TCM5 is also in line with observations of Simo et al. [15] who reported similar results in domestic animals of the same area. However, the amplification of nine samples from the DRC by this marker indicates that some genetic differences are probably due to mutations at the primer binding sites in trypanosomes circulating in villages of Fontem. This would mean that TCM5 seems to be an unsuitable genetic marker for subsequent investigations on the genetic characterization of the forest type of $T$. congolense. TCM3 neither amplified the Fontem samples nor those from the DRC. These results corroborate those obtained by Morrison et al. [14] and Simo et al. [15] and suggest TCM3 as a suitable marker for the differentiation of savannah and forest types of $T$. congolense. The variations in the size of alleles for each microsatellite locus corroborate results obtained by Morrison et al. [14] and Simo et al. [15] on T. congolense samples of Gambia and Cameroon respectively. The great disparity between the sizes of alleles observed here and for almost all the microsatellite data suggests that the microsatellite evolution within trypanosomes does not occur in a strictly stepwise manner.

Most alleles identified in this study were found in all villages. These alleles belong to major genotypes and 


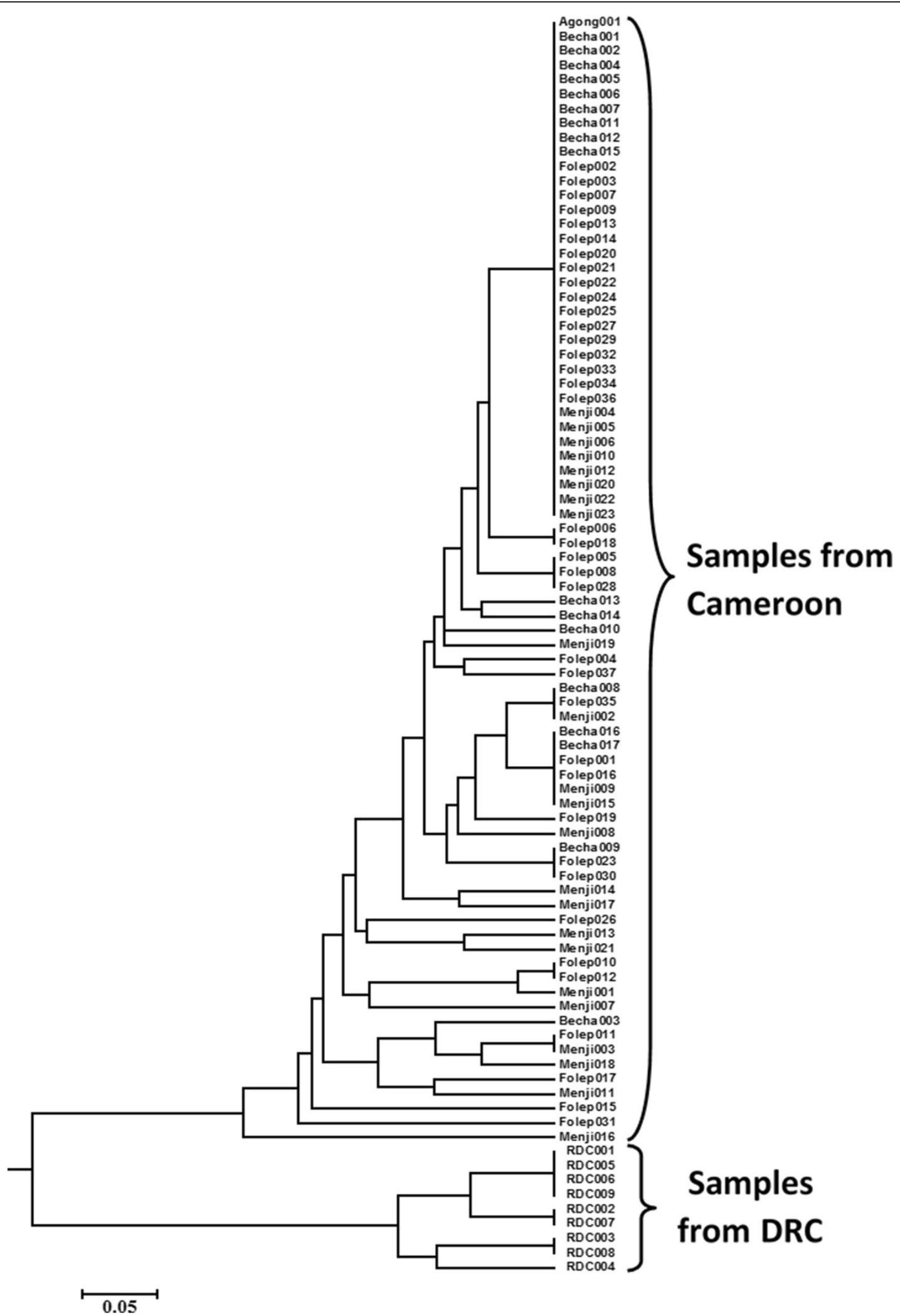

Figure 2 Dendrogram showing the genetic similarity between $T$. congolense forest circulating in tsetse flies of different villages of Fontem. 


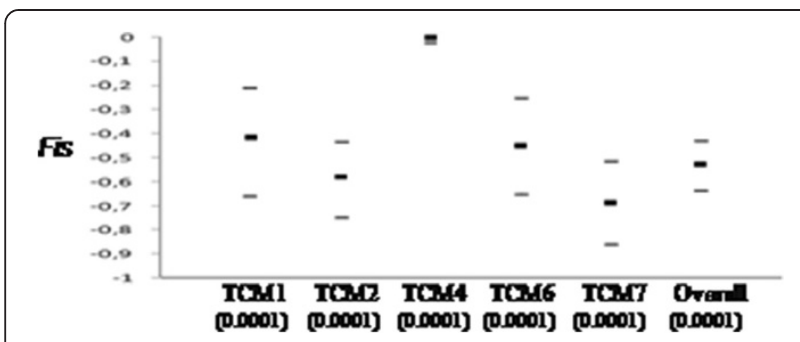

Figure 3 Individual fixation index (FIS) at Fontem for each locus and overall. For each locus, the 95\% confidence intervals were obtained by Jackknife over populations except for overall loci where it was obtained by bootstraps.

indicate the circulation of these major genotypes in all villages of Fontem. Beside these major genotypes, minor genotypes were also observed mainly in trypanosomes found in tsetse flies caught at Menji. These results are in line with those obtained in domestic animals of the same area [15]. For instance, the allele $180 \mathrm{bp}$ of TCM4 was identified in tsetse flies of Menji only. Recently, the same allele was identified in pigs of the same village, confirming the circulation of the same minor and major genotypes between animals and tsetse flies.

Both mixed and multiple (infection of different types of different species or subspecies of trypanosomes) infections were found in our study, corroborating the results of Morlais et al. [32] and Malele et al. [36] who have shown that infected tsetse flies from Cameroon and other zones of Africa frequently harbor more than one trypanosome species or subspecies. The distribution of alleles according to the year of capture shows that the number of new alleles has increased from 17 in 2007 to 21 in 2009. This increasing number of alleles could be explained by genetic exchanges, which may occur between different strains or mutations at some microsatellite loci. Indeed, many genotypes of the same species in the midguts of tsetse flies seem to be a principal cause of the appearance of new genotypes within species having mainly a clonal reproduction [37].

The population genetic analyses revealed low genetic variability between isolates of $T$. congolense forest that circulate in tsetse flies caught in the same village and in different villages of Fontem. This result is in line with results reported by Simo et al. [15] in domestic animals

Table $4 \boldsymbol{F}_{\mathrm{ST}}$ value between villages and the Democratic Republic of Congo

\begin{tabular}{llll}
\hline Villages & Folepi & Menji & DRC \\
\hline Bechati & $-0.0068^{*}$ & $-0.0009^{*}$ & 0.4212 \\
Folepi & & $0.0003^{*}$ & 0.4190 \\
Menji & & & 0.3911 \\
\hline
\end{tabular}

DRC: Democratic Republic of Congo; *P value not significant. of the same locality. The heterozygote excess (Table 4), as revealed by the $F_{\text {IS }}$ values (Figure 3 ), permit us to conclude in favor of a clonal reproduction of $T$. congolense. These results corroborate those of Tibayrenc et al. [13] and Simo et al. [15]. However, using the same microsatellite markers, Morrison et al. [14] demonstrated that mating apparently occurs in $T$. congolense, although it is not obligatory. There is, therefore, some controversy on the population genetics of $T$. congolense that requires further investigation in different areas of Africa in order to have a clearer understanding of the genetic structure of this parasite.

The analysis of the dendrogram in Figure 2 showed that the $T$. congolense sub-population of the DRC is genetically different from that of Cameroon. Indeed, the strains of the sub-population of DRC form one cluster while all strains of the sub-population of Fontem are grouped in another big cluster with low genetic distances between strains of DRC or strains of Fontem villages. However, important genetic distances were observed between the strains of DRC and those of Fontem, as illustrated by the dendrogram showing the genetic similarities between strains (Figure 2). The genetic differentiation between the samples of the DRC and those of Cameroon was strongly significant as shown by the $F_{\mathrm{ST}}$ value of $0.39(\mathrm{P}<0.0001)$. These results show a perfect sub-structuring between samples of the DRC and those of Cameroon. This important sub-structuration could be explained by the geographic distance between these two countries or between the sampling areas. There was no substructuration between different subpopulations of the forest type of $T$. congolense that circulates in tsetse flies of different villages of Fontem as shown by the low and non-significant difference between the $F_{\mathrm{ST}}$ values of different villages and different years of capture. This is highlighted by the heterogeneity in the distribution of samples in different nodes or clusters of the dendrogram (Figure 2) that illustrates genetic similarities between strains. The absence of sub-structuration within the populations of $T$. congolense that infect tsetse flies of different villages of Fontem suggests that the same genotypes or genotype families circulate in this area.

\section{Conclusion}

The results of this study have shown that the infection rates of $T$. congolense vary significantly between the populations of tsetse flies of different villages of Fontem as well as the years of capture. The microsatellite markers showed a low genetic variability within Fontem samples. Our results on the population genetics of $T$. congolense forest plead in favor of a predominant clonal reproduction of these parasites. No sub-structuring was observed between subpopulations of different villages of Fontem 
although with DRC samples, a perfect structuration was observed, certainly due to geographical distance between the two sampling areas.

\section{Additional files}

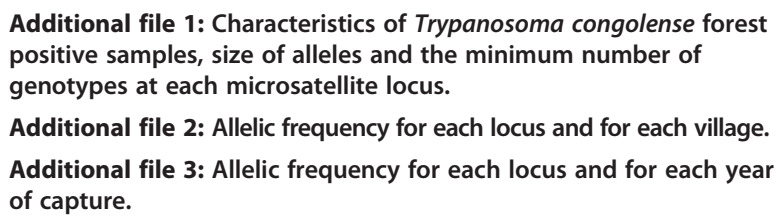

\section{Competing interests}

The authors declare that they have no competing interests.

\section{Authors' contributions}

GS participated in the conception of the study, design of experiments, collected field data and draft the manuscript. PSF performed molecular analyses and drafted the manuscript. TTTM helped in the population genetics analysis and the draft the manuscript. FN participated in the conception of the study, collected field data and helped to draft the manuscript. JRK helped to draft the manuscript. TA participated in the conception of the study and helped to draft the manuscript. All authors read and approved the final manuscript.

\section{Acknowledgements}

This study was supported by AIRES-SUD project n7003, UMR 177 IRD/CIRAD, the University of Yaoundé I and the University of Dschang.

\section{Author details}

'Molecular Parasitology and Entomology Unit, Department of Biochemistry, Faculty of Science, University of Dschang, Cameroon, PO Box 67, Dschang, Cameroon. ${ }^{2}$ Department of Animal Biology and Physiology, Parasitology and Ecology Laboratory, Faculty of Science, University of Yaoundé 1, P.O. Box 812 Yaoundé, Cameroon. ${ }^{3}$ Department of Biochemistry, Faculty of Science, University of Dschang, P.O. Box 67, Dschang, Cameroon. ${ }^{4}$ Faculty of Medicine and Biomedical Science, University of Yaoundé 1, P.O. Box 1364, Yaoundé, Cameroon.

Received: 17 April 2014 Accepted: 8 August 2014 Published: 20 August 2014

\section{References}

1. Steverding D: The history of African trypanosomiasis. Parasit Vectors 2008, 1:3. doi:10.1186/1756-3305-1-3.

2. Gros F: La mouche Tsé-Tsé. In Collection Les Saviors. 2004:36.

3. Mattioli R, Feldmann U, Hendrickx G, Wint W, Jannin J, Slingenbergh J: Tsetse and trypanosomiasis intervention policies supporting sustainable animal agricultural development. J Food Agr Environ 2004, 2:310-314

4. Hursey BS: The programme against African trypanosomiasis: aims, objectives and achievements. Parasitol 2001, 17:2-3.

5. Masumu J, Marcotty T, Geysen D, Geerts S, Vercruysse J, Dorny P, Van den Bossche P: Comparison of the virulence of Trypanosoma congolense strains isolated from cattle in a trypanosomiasis endemic area of eastern Zambia. Int J Parasitol 2006, 36:497-501.

6. Sigauque I, Van den Bossche P, Moiana M, Jamal S, Neves L: The distribution of tsetse (Diptera: Glossinidae) and bovine trypanosomoses in the Matutuine District, Maputo Province, Mozambique. Onderstepoort J Vet Res 2000, 67:167-172.

7. Simukoko H, Marcotty T, Phiri I, Geysen D, Vercruysse J, Van den Bossche P: The comparative role of cattle, goats and pigs in the epidemiology of livestock trypanosomosis on the plateau of Eastern Zambia. Vet Parasitol 2007, 147:231-238

8. Van Den Bossche P, Esterhuizen J, Nkuna R, Matjila T, Penzhorn B, Geerts S, Marcotty T: An update of the bovine trypanosomoses situation at the edge of Hluhiuwe-Imfolozi Park, Kwazulu-Natal Province, South Africa. Onderstepoort J Vet Res 2006, 73:77-79.
9. Cox AP, Tosas O, Tilley A, Picozzi K, Coleman P, Hide G, Welburn SC: Constraints to estimating the prevalence of trypanosome infections in East African zebu cattle. Parasit Vectors 2010, 3:82.

10. Simo G, Herder S, Njiokou F, Asonganyi T, Tilley A, Cuny G: Trypanosoma brucei s.l: characterization of stocks from Central Africa by PCR analysis of mobile genetic elements. Exp Parasitol 2005, 110:353-362

11. Koffi M, De Meeûs T, Bucheton B, Solano P, Camara M, Kaba D, Cuny G, Ayala FJ, Jamonneau V: Population genetics of Trypanosoma brucei gambiense, the agent of sleeping sickness in Western Africa. Proc Natl Acad Sci U S A 2009, 106:209-214.

12. Truc $P$, Tiouchichine ML, Cuny G, Vatunga G, Josenando T, Simo G, Herder S: Multiple infections of Trypanosoma brucei gambiense in blood and cerebrospinal fluid of human African trypanosomosis patients from Angola: consequences on clinical course and treatment outcome. Infect Genet Evol 2012, 12:399-402.

13. Tibayrenc M, Kjellberg F, Arnaud J, Oury B, Breniere SF, Dardé ML, Ayala FJ: Are eukaryotic microorganisms clonal or sexual? A population genetics vantage. Proc Natl Acad Sci U S A 1991, 88:5129-5133.

14. Morrison LJ, Tweedie A, Black A, Pinchbeck GL, Christley RM, Schoenefeld A, Hertz-Fowler C, MacLeod A, Turner CM, Tait A: Discovery of mating inthe major African livestock pathogen Trypanosoma congolense. PLoS One 2009, 4:e5564

15. Simo G, Fogue SP, Nijtchouang GR, Njiokou F, Kuiate JR, Cuny G, Asonganyi T: Identification and genetic characterization of Trypanosoma congolense in domestic animals of Fontem in the South-West region of Cameroon. Infect Genet Evol 2013, 18:66-73.

16. Masumu J, Marcotty T, Geerts $S$, Vercruysse J, Van den Bossche P: Cross-protection between Trypanosoma congolense strains of low and high virulence. Vet Parasitol 2009, 163:127-131.

17. Truc $P$, Tibayrenc M: Population genetic of Trypanosoma brucei in central Africa. Parasitol 1993, 106:137-149.

18. Simo G, Njitchouang GR, Njiokou F, Cuny G, Asonganyi T: Trypanosoma brucei sp: Microsatellite markers revealed high level of multiple genotypes in the mid-guts of wild tsetse flies of the Fontem sleeping sickness focus of Cameroon. Exp Parasitol 2011, 128:272-278.

19. Gouteux JP, Lancien J: The pyramidal trap for collecting and controlling tsetse flies (Diptera: Glossinidae). Comparative trials and description of new collecting techniques. Trop Med Parasitol 1986, 37:61-66.

20. Simo G, Mansinsa Diabakana P, Kande Betu Ku Mesu V, Zola Manzambi E, Ollivier G, Asonganyi T, Cuny G, Grébaut P: Human African Trypanosomiasis transmission, Kinshasa, Democratic Republic of Congo (DRC). Emerg Infect Dis 2006, 12:1968-1970.

21. Masiga DK, Smyth AJ, Hayes P, Bromidge TJ, Gibson WC: Sensitive detection of trypanosomes in tsetse flies by DNA amplification. Int $J$ Parasitol 1992, 22:909-918.

22. Majiwa PA, Thatthi R, Moloo SK, Nyeko JH, Otieno LH, Maloo S: Detection of trypanosome infections in the saliva of tsetse flies and buffy-coat samples from antigenaemic but aparasitaemic cattle. Parasitol 1994, 108:313-322.

23. Herder S, Simo G, Nkinin S, Njiokou F: Identification of trypanosomes in wild animals from southern Cameroon using the polymerase chain reaction (PCR). Parasite 2002, 9:345-349.

24. Wright $\mathrm{S}$ : The interpretation of population structure by F-statistics with special regard to systems of mating. Evolution 1965, 19:395-420.

25. Weir BS, Cockerham CC: Estimating F-statistics for the analysis of population structure. Evolution 1984, 38:1358-1370.

26. Goudet J: Fstat (ver. 2.9.4): a program to estimate and test population genetics parameters. 2003. Available from http://www2.unil.ch/popgen/ softwares/ Updated from Goudet [1995].

27. Goudet J: FSTAT (Version 1.2): a computer program to calculate F-statistics. $J$ Hered 1995, 86:485-486.

28. Kumar S, Tamura K, Nei M: MEGA3: integrated software for molecular evolutionary genetics analysis and sequence alignment. Brief Bioinform 2004, 5:150-163.

29. Cavalli-Sforza LL, Edwards AWF: Phylogenetic analysis: models and estimation procedures. Am J Hum Genet 1967, 19:233-257.

30. Dieringer D, Schlotterer C: Microsatellite analyser (MSA): a platform independent analysis tool for large microsatellite data sets. Mol Ecol Notes 2003, 3:167-169.

31. Felsenstein J: PHYLIP (Phylogeny Inference Package) version 3.69. Distributed by the author. In $\bigotimes$ University of Washington, Seattle: Department of Genetics; 1993. 
32. Morlais I, Grebaut P, Bodo JM, Djoha S, Cuny G: Characterization of trypanosome infections by polymerase chain reaction (PCR) amplification in wild tsetse flies in Cameroon. Parasitol 1998, 116:47-54.

33. Nimpaye H, Njiokou F, Njine T, Njitchouang GR, Cuny G, Herder S, Asonganyi T, Simo G: Trypanosoma vivax, T. congolense forest type and $T$. simiae: prevalence in domestic animals of sleeping sickness foci of Cameroon. Parasite 2011, 18:171-179.

34. Simo G, Asonganyi T, Nkinin SW, Njiokou F, Herder S: High prevalence of Trypanosoma bruceigambiense group 1 in pigs from the Fontem sleeping sickness focus in Cameroon. Vet Parasitol 2006, 139:57-66.

35. Bengaly Z, Sidibe I, Ganaba R, Desquesnes M, Boly H, Sawadogo L: Comparative pathogenicity of three genetically distinct types of Trypanosoma congolense in cattle: clinical observations and haematological changes. Vet Parasitol 2002, 108:1-19.

36. Malele I, Graske L, Knight C, Ferris V, Njiru Z, Zamilton P, Lehane M, Gibson $W$ : The use of specific and genetic primers to identify trypanosomes infectious of wild tsetse flies in Tanzania by PCR. Infect Genet Evol 2003, 3:271-279.

37. Peacock L, Ferrisa V, Bailey M, Gibsona W: Intraclonal mating occurs during tsetse transmission of Trypanosoma brucei. Parasit Vectors 2009, 2: 43:12. doi:10.1186/1756-3305-2-43.

doi:10.1186/1756-3305-7-385

Cite this article as: Simo et al:: Population genetics of forest type of

Trypanosoma congolense circulating in Glossina palpalis palpalis of Fontem in the South-West region of Cameroon. Parasites \& Vectors 2014 7:385.

\section{Submit your next manuscript to BioMed Central and take full advantage of:}

- Convenient online submission

- Thorough peer review

- No space constraints or color figure charges

- Immediate publication on acceptance

- Inclusion in PubMed, CAS, Scopus and Google Scholar

- Research which is freely available for redistribution 